before receiving $\mathrm{ET}$, providing a possible explanation as to why no neuroprotective benefit of ET was noted in these trials.

Original article Suzuki S et al. (2007) Timing of estrogen therapy after ovariectomy dictates the efficacy of its neuroprotective and antiinflammatory actions. Proc Natl Acad Sci USA 104: 6013-6018

\section{Perfusion-weighted MRI predicts cerebral hyperperfusion after carotid endarterectomy}

A small subset of patients who undergo carotid endarterectomy (CEA) develop cerebral hyperperfusion syndrome, a serious complication with a poor prognosis. In a recent study, Fukuda et al. have investigated whether the quantitative measurement of preoperative cerebral blood volume (CBV) by perfusionweighted MRI could be used to predict postCEA cerebral hyperperfusion in patients with unilateral internal carotid artery stenosis. Hyperperfusion was assessed by CT, and was defined as an at least twofold increase in ipsilateral cerebral blood flow (CBF) relative to preoperative measurements.

In total, 15 of 70 patients had elevated preoperative CBV (relative to control values obtained from 10 healthy individuals). Of these 15 patients, 7 demonstrated hyperperfusion immediately after CEA; by contrast, none of the 55 patients with normal CBV values exhibited postoperative hyperperfusion. CBV correlated significantly with post-CEA increases in CBF (coefficient of correlation $r=0.785$; $P<0.0001)$, and logistic regression identified CBV as the only independent predictor of postoperative hyperperfusion. Five of the seven patients who had hyperperfusion immediately after CEA had normal CBF on the third day after surgery; the remaining two patients developed hyperperfusion syndrome. Preoperative CBV values did not, however, differ between the two patients who developed hyperperfusion syndrome and the five in whom hyperperfusion quickly resolved.

The authors conclude that perfusionweighted MRI measurement of pre-CEA CBV could identify patients at risk for hyperperfusion syndrome. They note, however, that this approach might not accurately measure
CBV in patients with contralateral internal carotid artery steno-occlusive disease.

Original article Fukuda T et al. (2007) Prediction of cerebral hyperperfusion after carotid endarterectomy using cerebral blood volume measured by perfusion-weighted MR imaging compared with single-photon emission CT. AJNR Am J Neuroradiol 28: 737-742

\section{Disruption to brain circuitry linked to spatial neglect}

Spatial neglect is a common syndrome following right hemisphere stroke. Patients with spatial neglect have trouble perceiving and responding to stimuli on the contralesional side of the body. Conventionally, this syndrome has been explained in terms of damage to regions of the brain directly affected by stroke. Now, researchers from the Washington University School of Medicine report an association between spatial neglect and a breakdown in communication between two largely separate brain areas that control attention.

Using functional connectivity MRI, the researchers examined functional brain circuitry in 11 patients with spatial neglect following stroke, and in 24 healthy individuals. Patients were scanned within a month of stroke and again more than 6 months after stroke. During the brain scan, study participants were asked to indicate whether they saw an asterisk on the left or right side of a video screen. Patients with spatial neglect had significantly more misses than did controls at both screenings $(P=0.0002$ and $P=0.01)$.

Scanning revealed that connectivity in the directly stroke-damaged ventral frontoparietal area was disrupted. Notably, scanning also revealed disruption to connectivity in the structurally intact dorsal frontoparietal area. In addition, the researchers found a correlation between the degree of disconnection within and between the dorsal and ventral networks and the severity of spatial neglect. The authors conclude that functional connectivity is required for normal brain function, and that connectivity between two brain regions can be disrupted even in the absence of direct damage to both areas.

Original article He BJ et al. (2007) Breakdown of functional connectivity in frontoparietal networks underlies behavioral deficits in spatial neglect. Neuron 53: 905-918 